\title{
Mercatorkarte und hyperbolische Geometrie
}

Hansklaus Rummler

\begin{abstract}
Hansklaus Rummler studierte Mathematik an der Westfälischen Wilhelms-Universität Münster und an der Universität Freiburg in der Schweiz, wo er auch 1968 promovierte und seit dem Wintersemester 1980/81 als Assistenzprofessor bzw. Professeur associé unterrichtet. Seine mathematischen Interessen betreffen die Beziehungen zwischen Analysis und Geometrie.
\end{abstract}

\section{Die Mercatorkarte}

Diese winkeltreue Abbildung der Erdoberfläche auf einen Zylinder, der dann aufgeschnitten und in der Ebene abgerollt wird, wurde 1569 von Mercator, wie sich Gerhard Kremer (1512-1594) latinisiert nannte, in Duisburg angefertigt. Er hatte bemerkt, daß auf einer Plattkarte, bei der das Koordinatennetz der geographischen Länge und Breite einfach als kartesisches Koordinatennetz in der Ebene wiedergegeben wird, die Breitenkreise mit zunehmender Entfernung vom Äquator zunehmend vergrößert abgebildet werden und daß man dieselbe Verzerrung auf die Meridiane anwenden muß, wenn man eine winkeltreue Karte haben will. Fig. 1 zeigt so eine Mercatorkarte; der Abstand zwischen zwei Gitterlinien beträgt jeweils $15^{\circ}$.

Der Cambridger Mathematiker Edward Wright publizierte im Jahre 1600 „A Chart of the World on Mercator's Projection“, nachdem er in seinem 1599 erschienenen Buch The Correction of Certain Errors in Navigation das Rezept angegeben hatte:

Faßt man die geographischen Koordinaten eines Punktes der Sphäre als kartesische Koordinaten eines Punktes der Ebene auf, so erhält man eine Plattkarte der Sphäre. Durch geschickte Streckung der Meridiane erhält man daraus die winkeltreue Mercatorkarte. Ganz analog erhält man bei Verwendung sogenannter Fermikoordinaten für die hyperbolische Ebene eine Plattkarte derselben, die sich ebenfalls durch geschickte Verzerrung der „Meridiane“ zu einer winkeltreuen Karte abändern läßt. Die dazu notwendige Verkürzung der „Meridiane“ wird gerade von der Umkehrung der Funktion geleistet, die die Streckung der Meridiane für die gewöhnliche Mercatorkarte beschreibt. Aus dieser winkeltreuen Karte der hyperbolischen Ebene erhält man mit konformen Standardabbildungen aus der Funktionentheorie die Poincaréschen Modelle, die ja ebenfalls winkeltreu sind. 


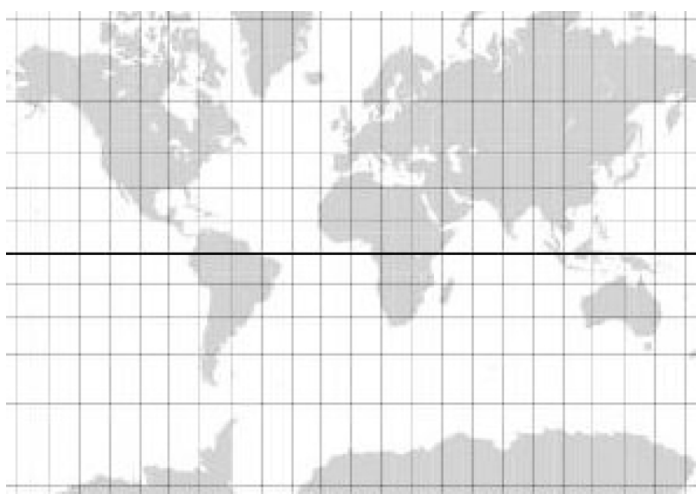

Fig. 1

\begin{abstract}
„The parts of the meridian at every poynt of latitude must needs increase with the same proportion wherewith the Secantes or hypotenusae of the arke, intercepted betweene those pointes of latitude and the aequinoctiall do increase ... by perpetuall addition of the Secantes answerable to the latitudes of each point ... we may make a table which shall shew the sections and points of latitude in the meridians of the nautical planisphaere: by which sections, the parallels are to be drawne." (Siehe [4], S. 701.)
\end{abstract}

Wright publizierte auch gleich die Tafel mit den Ergebnissen seiner ,perpetuall addition": sie enthielt die Summen der Sekanten, d.h. der Kehrwerte der Cosinus, in Minutenschritten von 0 bis $\varphi$ für Winkel $\varphi$ zwischen $0^{\circ}$ und $75^{\circ}$. Diese Tafel enthält also Approximationen des Integrals

$$
v=\int_{0}^{\beta} \frac{d \varphi}{\cos \varphi}
$$

durch das wir heute die Mercatorbreite $v(\beta)$ beschreiben. Ihre Umkehrung $\beta(v)$ ist die Gudermannfunktion (siehe dazu auch [1]); wir werden ihr weiter unten wieder begegnen. Fig. 2 zeigt den Graphen dieser Beziehung zwischen der geographischen Breite $\beta$ auf der Sphäre und der Höhe $v$ auf dem Zylinder, sowie einige der zahlreichen Formeln, die sie beschreiben. (Der Nachweis der Äquivalenz dieser Formeln liefert gute Übungsaufgaben zur Analysis der trigonometrischen und hyperbolischen Funktionen!)

Die Mercatorprojektion von der Sphäre auf den Zylinder, oder noch einfacher ihre Umkehrung, läßt sich natürlich als konforme Abbildung recht elegant mit den Mitteln der Funktionentheorie beschreiben:

Die Exponentialabbildung kann wegen ihrer Periodizität als Abbildung des Zylinders auf die gelochte Ebene $\mathbb{C}^{*}$ aufgefaßt werden, die wir anschließend mit der stereographischen Projektion vom Nordpol aus auf die Sphäre abbilden. Das Resultat ist genau die Umkehrung der Mercatorprojektion, und der Identitätssatz der Funktionentheorie liefert uns auch gleich die Eindeutigkeit: Es ist die einzige winkeltreue Karte, die den Äquator längentreu als Kreis des Zylinders bzw. als Gerade darstellt. (Siehe [3], S. 259, Exercise 14.) 


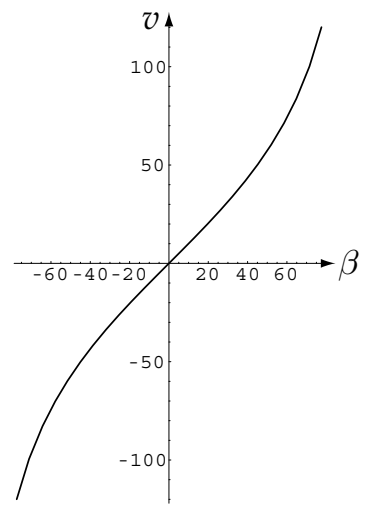

$$
\begin{aligned}
\tanh \frac{v}{2} & =\tan \frac{\beta}{2}, \\
\tanh v & =\sin \beta, \\
\sinh v & =\tan \beta, \\
v & =\ln \tan \left(\frac{\pi}{4}+\frac{\beta}{2}\right), \\
\beta & =\frac{\pi}{2}+2 \arctan \mathrm{e}^{v} .
\end{aligned}
$$

Fig. 2

\section{Eine Plattkarte der hyperbolischen Ebene}

Wir bezeichnen mit $\mathbb{E}$ die ,abstrakte“ hyperbolische Ebene, aufgefaßt als eine orientierte zweidimensionale Riemannsche Mannigfaltigkeit der konstanten Gaußschen Krümmung $K=-1$. Um in ihr Koordinaten einzuführen, fixieren wir eine orientierte (hyperbolische!) Gerade $g$ sowie einen Punkt $O$ auf dieser Geraden. Einem beliebigen Punkt $P \in \mathbb{E}$ können wir dann wie folgt Koordinaten $u(P)$ und $v(P)$ zuordnen:

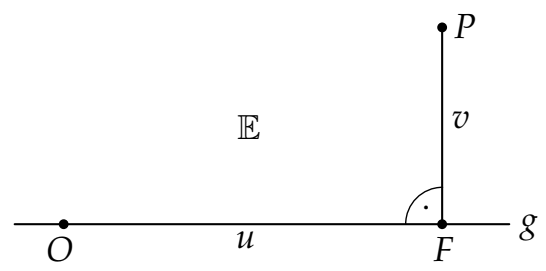

Fig. 3

Ist $F$ der Fußpunkt des Lots von $P$ auf $g$, so setzen wir

$$
u(P):= \pm \operatorname{dist}(O, F), \quad v(P):= \pm \operatorname{dist}(F, P),
$$

wobei wir das Vorzeichen durch die Orientierung festlegen, also $u>0$, falls die gerichtete Strecke von $O$ nach $F$ die Orientierung von $g$ repräsentiert, und $v(P)>0$, wenn $P$ im Sinne der Orientierungen von $g$ und $\mathbb{E}$,links“ von $g$ liegt.

Die so definierten Koordinaten, die sich in derselben Weise auf jeder orientierten Fläche in der Umgebung einer Geodätischen einführen lassen, werden als Fermikoordinaten bezeichnet. (Siehe [2], S. 103-104.) In unserem Falle liefern sie einen Diffeomorphismus der ganzen hyperbolischen Ebene $\mathbb{E}$ auf den Euklidischen Raum $\mathbb{R}^{2}$, also eine globale Karte der hyperbolischen Ebene. In dieser Karte ist die ausgezeichnete hyperbolische Gerade $g$ als Euklidische Gerade dargestellt, und zwar längentreu. Dasselbe gilt für die $g$ senkrecht schneidenden Geraden, deren Bilder in der Karte das Bild von $g$ ebenfalls senkrecht schneiden. Dagegen stellen die zum Bild von $g$ parallelen Geraden des $\mathbb{R}^{2}$ 
keine hyperbolischen Geraden dar, sondern Äquidistanzlinien, d.h. Linien konstanten Abstandes von der Geraden g.

Diese Karte spielt für die Darstellung der hyperbolischen Ebene $\mathbb{E}$ die gleiche Rolle, wie sie die Plattkarte für die Darstellung der Sphäre $\mathbb{S}$ spielt: Dort wird der Äquator als Gerade dargestellt, und die dazu parallelen Geraden der Karte entsprechen ebenfalls den Kurven konstanten Abstandes vom Äquator, nämlich den Breitenkreisen.

\section{Eine Mercatorkarte der hyperbolischen Ebene}

Wie Mercator die Plattkarte der Erde zu einer winkeltreuen Karte abänderte, indem er die Distanz zwischen den Bildern der Breitenkreise geschickt streckte, so wollen wir dasselbe mit der Plattkarte der hyperbolischen Ebene machen. Dazu müssen wir erst einmal die Längenmessung bei Verwendung der Fermikoordinaten untersuchen:

Da die Geraden $\{u(P)=$ const $\}$ längentreu in den $\mathbb{R}^{2}$ abgebildet werden, brauchen wir nur noch die Längenmessung der Linien $\{v(P)=$ const $\}$ anzuschauen. Dazu betrachten wir ein Viereck, bei dem die Grundseite $A B$ auf der Geraden $g$ liegt, während $C$ und $D$ von $g$ den Abstand $v$ haben und $A D$ und $B C$ senkrecht auf $g$ stehen. (Siehe Fig. 4.) Die Seite $C D$ ist dabei keine Gerade, sondern eine Äquidistanzlinie: alle Punkte dieser Seite haben von $g$ denselben Abstand $v$.

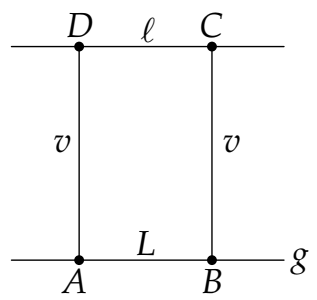

Fig. 4

Wir betrachten jetzt $v$ als variabel und untersuchen bei fester Länge $L$ der Grundseite $A B$ die beiden Funktionen

$$
\ell(v):=\text { Länge der Seite } C D \text { und } F(v):=\text { Fläche des Vierecks } A B C D \text {. }
$$

Die erste Fundamentalform hat in den Fermikoordinaten $u, v$ die Matrix

$$
\left(\begin{array}{cc}
1 & 0 \\
0 & (\partial s / \partial u)^{2}
\end{array}\right)
$$

wobei $s$ die Bogenlänge der Linien $v=$ const ist. Daraus erhält man für das Flächenelement den Ausdruck $d F=(\partial s / \partial u) d u d v$ und daher

$$
F^{\prime}(v)=\int_{C}^{D} \frac{\partial s}{\partial u} d u=\ell(v)
$$

Die geodätische Krümmung $\kappa$ der Seite $C D$, orientiert von $C$ nach $D$, ist ein Maß für die Längenänderung der Seite, wenn wir sie in Richtung des Normalenfeldes verschieben; 
genauer gilt:

$$
\ell^{\prime}(v)=\int_{C}^{D} \kappa d s
$$

Andererseits gilt für das Viereck die Gauß-Bonnet-Formel:

$$
\int_{C}^{D} \kappa d s+4 \cdot \frac{\pi}{2}+\int_{A B C D} K d o=2 \pi,
$$

woraus wegen $K \equiv-1$ schließlich folgt:

$$
\ell^{\prime}(v)=F(v) .
$$

Mit den Anfangsbedingungen $\ell(0)=L:=$ Länge der Seite $A B$ und $F(0)=0$ erhält man sofort die Lösung von (1) und (2), wobei uns hier nur die Funktion $\ell(v)$ interessiert:

$$
\ell(v)=L \cosh v .
$$

Fassen wir unsere Ergebnisse zusammen, so können wir sagen, daß in der Karte die Euklidische Längenmessung auf den Linien $u=$ const korrekt ist, daß sie aber auf den Linien $v=$ const zu klein ausfällt: die in der Karte gemessenen Längen müssen mit dem Faktor $\cosh v$ multipliziert werden. Wenn wir also dieselbe Korrektur anwenden wollen wie Mercator und Wright, müssen wir die Linien $u=$ const in der Karte entsprechend verkürzen. Die Linie $v=v_{0}$ darf von der Linie $v=0$ nur den Abstand

$$
\beta_{0}=\int_{0}^{v_{0}} \frac{d v}{\cosh v}=2 \arctan \tanh \frac{v_{0}}{2}
$$

haben. Unsere Karte der hyperbolischen Ebene wird also genau dann winkeltreu, wenn wir die Koordinate $v$ durch die Koordinate $\beta$ ersetzen, wobei $\beta(v)$ die Gudermannfunktion ist. Die Rollen von $\beta$ und $v$ sind also gegenüber dem Fall der Sphäre gerade vertauscht!

\section{Poincaré-Modelle der hyperbolischen Ebene}

Unsere winkeltreue Karte stellt die hyperbolische Ebene $\mathbb{E}$ in einem unendlich langen Streifen der Breite $\pi$ dar. Den wollen wir jetzt noch einer kleinen funktionentheoretischen Nachbehandlung unterziehen, um eine ,handlichere“ Karte zu bekommen, indem wir ihn konform auf die obere Halbebene $\mathbb{H}$ abbilden:

Diese Abbildung erhalten wir durch

$$
(u, \beta) \longmapsto \mathrm{ie}^{u+\mathrm{i} \beta} .
$$

Kombinieren wir sie mit unserer Kartenprojektion, so erhalten wir insgesamt die folgende Kartenprojektion $\Phi: \mathbb{E} \rightarrow \mathbb{H}$ :

$$
\Phi(P)=\mathrm{ie}^{u(P)+\mathrm{i} \beta(v(P))} .
$$

Die ausgezeichnete Gerade $g=\{P \in \mathbb{E} ; v(P)=0\}$ wird also auf die positive imaginäre Halbachse abgebildet, wobei der Ursprung $O$ nach i geht. Die Linie $\left\{P \in \mathbb{E} ; v(P)=v_{0}\right\}$ wird auf die Halbgerade $\left\{r \mathrm{e}^{\mathrm{i} \beta\left(v_{0}\right)} ; r>0\right\}$ abgebildet.

Das ist natürlich das bekannte Poincarésche obere Halbebenenmodell der hyperbolischen Ebene. Wir haben es hier rein geometrisch erhalten, ohne die Isomorphie der Gruppe 
der orientierungserhaltenden Isometrien von $\mathbb{E}$ mit derjenigen der Automorphismen von $\mathbb{H}$ zu verwenden. Der Ehrlichkeit halber sei aber erwähnt, daß die Erkenntnis, daß $\mathbb{E}$ eine Fläche konstanter Gaußscher Krümmung $K=-1$ ist, auch nicht ganz einfach zu erhalten ist, obwohl das natürlich bereits Johann Heinrich Lambert geahnt hatte, der 1766 in seiner „Theorie der Parallellinien“ schrieb: „Ich sollte daraus fast den Schluß machen, die dritte Hypothese (d.h. diejenige, daß in einem geradlinigen Viereck mit drei rechten Winkeln der vierte kleiner als $90^{\circ}$ ist,) komme bey einer imaginären Kugelfläche vor." Selbst ohne diese Aussage präzisieren zu können, wäre er vermutlich in der Lage gewesen, die von uns verwendete Formel für die Länge $\ell(v)$ zu finden.

Wenn wir noch die Cayleytransformation $z \mapsto \frac{z-\mathrm{i}}{z+\mathrm{i}}$ anwenden, um von der oberen Halbebene $\mathbb{H}$ zur Einheitskreisscheibe $\mathbb{D}$ überzugehen, so erhalten wir die Kartenprojektion $\Psi: \mathbb{E} \rightarrow \mathbb{D}$, die unter Verwendung der Fermikoordinaten $u, v$ und der Gudermannfunktion $\beta(v)$ durch

beschrieben wird.

$$
\Psi(P)=\frac{\mathrm{e}^{u(P)+\mathrm{i} \beta(v(P))}-1}{\mathrm{e}^{u(P)+\mathrm{i} \beta(v(P))}+1}
$$

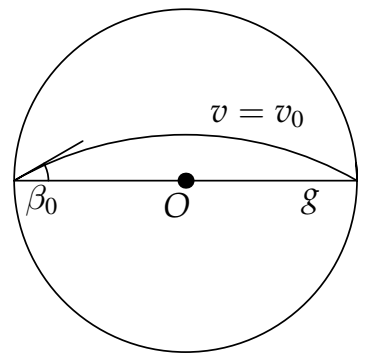

Fig. 5

Die ausgezeichnete Gerade $g$ wird dabei auf das Intervall ]-1, 1[ abgebildet, der Ursprung $O$ auf 0 und die Linie $\left\{v(P)=v_{0}\right\}$ auf einen Kreisbogen zwischen -1 und +1 , der die reelle Achse dort unter dem Winkel $\beta_{0}=\beta\left(v_{0}\right)$ schneidet. (Siehe Fig. 5.)

\section{Literatur}

[1] Greenberg, B.S.: http://www.beanpaste.com/BSG/mercator.html

[2] Küchel, W.: Differentialgeometrie, Vieweg, 1999.

[3] Needham, T.: Visual Complex Analysis, Clarendon Press, 1997.

[4] Tuchinsky, Ph.M.: Mercator's World Map and the Calculus, UMAP modules - Tools for Teaching, Birkhäuser, 1981, 681-727.

[5] Weimer, N.: http://www.ualberta.ca/ norris/navigation/Mercator.html

Hansklaus Rummler

Universität Freiburg

Departement für Mathematik

Chemin du Musée 23

CH-1700 Fribourg, Schweiz

e-mail: hansklaus.rummler@unifr.ch 